\title{
A comparative study of anode properties of two ordered mesoporous carbons with tailored crystalline structure for lithium ion batteries
}

\author{
Jianguo Cao ${ }^{1,2}$, Zhaohui Hou ${ }^{2, \text { a }}$, Fanyan Zeng ${ }^{1,2}$, Chongyong Ge ${ }^{1,2}$, Yafei \\ Kuang ${ }^{1, b}$ \\ ${ }^{1}$ College of Chemistry and Chemical Engineering, Hunan University, Hunan, Changsha 410082, \\ PR China \\ ${ }^{2}$ College of Chemistry and Chemical Engineering, Hunan Institute of Science and Technology, \\ Hunan, Yueyang 414006, PR China \\ azhqh96@163.com(Z. H. Hou), byafeik@163.comT (Y. F. Kuang)
}

Keywords: Lithium ion batteries; Ordered mesoporous carbon; Tailored crystalline structure; Anode properties.

\begin{abstract}
Ordered mesoporous carbons (C-1 and C-2) with tailored pore size and defined crystal orientation are prepared by liquid crystal template carbonization method using AR-mesophase pitch as carbon precursors and SBA-15 as template. The topography, pore/crystalline structure are extensively characterized via high-resolution transmission electron microscope (HR-TEM), $\mathrm{N}_{2}$ adsorption/desorption and powder X-ray diffraction (XRD). The electrochemical properties of C-1 and $\mathrm{C}-2$ as anode materials for lithium ion batteries (LIBs) are examined by galvano-statically charge/discharge and electrochemical impedance spectroscopy (EIS). Materials characterization shows that the carbons prepared in this work replicate the ordered mesoporous structure of the SBA-15 and possess tailored crystalline structure with graphene layers perpendicular to the axial of pore walls. The carbons both display preferable anode performance for LIBs. However, C-2 with thicker pore wall exhibits better properties than $\mathrm{C}-1$ with larger pore size. Possible reasons for different properties are the influence of the pore structure and wall thickness of as-prepared mesoporous carbons.
\end{abstract}

\section{Introduction}

Owing to their high energy density, high output voltage and environmental friendliness relative to traditional batteries, LIBs are widely applied in portable electronic devices such as mobile phones, lap-tops and camcorders in recent years. However, for electric vehicles (EVs) and hybrid electric vehicles (HEVs) applications, LIB's inherent low power density circumvents their usage [1]. Therefore, increasing interest has been paid to the LIB materials with dramatically anode properties.

Currently, graphite is the most popular anode material for LIBs. However, the low lithium storage capacity (theoretically $372 \mathrm{mAh} \mathrm{g}^{-1}$ ), slow solid state diffusion coefficient of $\mathrm{Li}^{+}$and long $\mathrm{Li}^{+}$ diffusion distance are the key factors affecting application of graphite for LIBs. Nanostructured carbon materials, Especially mesoporous carbon materials have attracted a great deal of attention and exhibit enhanced performance for LIBs owing to their unique features, such as the small size, large contact area and short solid state diffusion distance [2]. It is a good alternative to employ mesoporous materials as the active intercalation host for $\mathrm{Li}^{+}$. Mesoporous carbons $[3,4]$, especially templated carbon [5,6,7] have displayed superior rate performance for $\mathrm{Li}^{+}$detercalation/ intercalation.

Recently, Bruce et al. [8] found that the pore size and wall thickness are the important influence factors to the rate performance of mesoporous $\beta-\mathrm{MnO}_{2}$ series as anode materials. The possible reason is that $\beta-\mathrm{MnO}_{2}$ possesses different pore size and wall thickness while preserving the same highly crystalline structure of within the walls [9]. Thus, we conjecture whether there exists the same influence on the anode properties of mesoporous carbons. Nowadays, mesoporous carbons that possess the tailored crystalline structure may be obtained by liquid crystal template carbonization method [10,11]. It is just that the melted liquid-crystal polycyclic aromatic hydrocarbons (PAHS) could self-assemble on the surface of some substrates at different angle, which provides a possible 
way to control the crystalline structure or the accumulation mode of graphene layers through molecular engineering.

In this work, we fabricated two ordered mesoporous carbons with tailored pore size $(\mathrm{C}-1$ and C-2) using the liquid crystal template carbonization method. The anode properties of the prepared carbons for LIBs are investigated comparatively.

\section{Experimental}

Ordered mesoporous silica SBA-15 (Tongji University) was used as template. AR-mesophase pitch (Mitsubishi Chemical Company), with average molecular weight of 2000 Dalton, and fusion point of $300{ }^{\circ} \mathrm{C}$, was used as carbon precursor. An assumption was made that, at the melting point, liquid-crystal AR-mesophase pitch could fully impregnate into the nanochannels of SBA-15 templates by capillarity [12]. Based on the density of AR-mesophase pitch and the total pore volume of SBA-15 templates, the calculated quantity of mesophase pitch powders that was needed to fill the pore volume of SBA-15 template was named as M1 and the experimental amount was denoted as M2.

The precursor and template were mixed sufficiently by a mass ratio of $\mathrm{M} 1: \mathrm{M} 2=1: 0.4$, followed by heating at $300^{\circ} \mathrm{C}$ with a heating rate of $4{ }^{\circ} \mathrm{C} \mathrm{min}{ }^{-1}$ and held for $7 \mathrm{~h}$ in tube furnace under argon atmosphere (99.99\%). At this condition, the melted liquid crystal mesophase pith could infiltrate into the template spontaneously by capillary force. Subsequently the temperature was increased to $900{ }^{\circ} \mathrm{C}$ with a heating rate of $4{ }^{\circ} \mathrm{C} \mathrm{min}^{-1}$ and kept for $2 \mathrm{~h}$ to fully carbonize AR-mesophase pitch. After cooling to room temperature, SBA-15 template was removed by in $10 \%$ HF under stirring. Finally, the resulted material was washed several times with deionized water, and dried at $100{ }^{\circ} \mathrm{C}$ for $24 \mathrm{~h}$ in an air oven. As-obtained sample was denoted as C-1. C-2 was also prepared with M1:M2 ratio of $1: 0.8$.

High-resolution transmission electron microscope (HR-TEM) (JEOL-3100, Japan) operated at $200 \mathrm{KV}$ was used for observing the morphologies and structures of SBA-15 and as-prepared carbons. Brunauer-Emmett-Teller (BET) surface area and pore structure of materials were measured by the N2 sorption isotherm analyzer (BECKMAN COULTER SA-3100, USA). The crystalline structure of as-prepared carbons was analyzed by X-ray diffraction (XRD) diffractometer (Bruker D8-Advanced, $40 \mathrm{kV}, 40 \mathrm{~mA}$ and $\mathrm{Cu} \mathrm{K} \alpha$ radiation).

Fabrication of working electrodes was carried out as follows. Synthesized carbon, acetylene black and poly (vinylidene difluoride) (PVDF) binder were mixed with a weight ratio of 80: 5: 15 to form a slurry. Then the slurry was coated on copper foil and dried at $120{ }^{\circ} \mathrm{C}$ for $24 \mathrm{~h}$ in a drying oven. Carbon foil was punched to disks with $14 \mathrm{~mm}$ diameter, and then pressed at $5 \mathrm{Mpa}$ about before using as working electrode.

Electrochemical performance measurements were performed using 2032 coin-type cells. Lithium sheet was used as the counter and reference electrode. $1 \mathrm{M} \mathrm{LiPF}_{6}$ solution in a 1:1 (V/V) mixture of ethylene carbonate (EC) + dimethyl carbonate (DMC) and porous polypropylene membrane was used as electrolyte and cell separator respectively. The cells were assembled in glove box filled with high purity $\mathrm{Ar}_{2}$ and kept at open circuit for $24 \mathrm{~h}$. Electrochemical impedance spectroscopy (EIS) was measured on electrochemical workstation (CHI 660B) in the frequency range of $1 \mathrm{mHz}-100 \mathrm{kHz}$. The cells were galvano-statically charged (intercalation) - discharged (deintercalation) in the voltage range of $0.005-2.0 \mathrm{~V}\left(\mathrm{vs} . \mathrm{Li}^{+} / \mathrm{Li}\right)$ on battery program-control test system (PCBT-100-8B, Wuhan Lisun Power Sources Co.,Ltd). The first three charge-discharge profiles were measured at the current density of $35 \mathrm{~mA} \mathrm{~g}^{-1}$ and the galvanostatic cycles were obtained at the current density of $70,350,1050,1750,3500$ and $4200 \mathrm{~mA} \mathrm{~g}^{-1}$, respectively.

\section{Results And Discussion}

Fig. 1 presents HR-TEM images of template and as-prepared carbons. It can be seen from Fig. 1 (a) and (b), C-1 replicates the ordered 2D symmetrical mesostructure of SBA-15. Images taken from (001) direction of C-1 and C-2 are shown in Fig. 1 (c) and (e), respectively. It can be seen that 
carbons consisted of nanofibers are aligned into bundle. The external diameter of unit fiber is estimated to be about $7.5 \mathrm{~nm}$, conforming to that of the pore diameter of SBA-15. Fig. 1 (d) and (f) exhibit that the graphene layers of C-1 and C-2 are perpendicular to the long axis of pore walls. Similar to platelet carbon nanofibers, many loops (8-10 carbon layers inter-connected to form a loop), which are parallel to the axial direction of nanofibers, are formed at the edge of graphene layers $[13,14]$. PAHS in the melted liquid crystal could possibly anchor on the internal surface of silica template, which is unaffected by carbonization process. As a result, the tailored crystalline structure is obtained $[15,16]$.
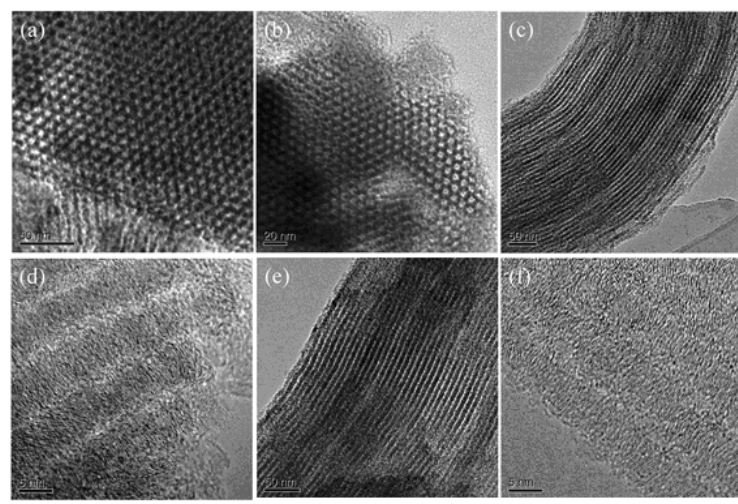

Fig. 1. HR-TEM images of SBA-15, C-1 and C-2: (a) SBA-15; (b, c, d) C-1 and (e, f) C-2.
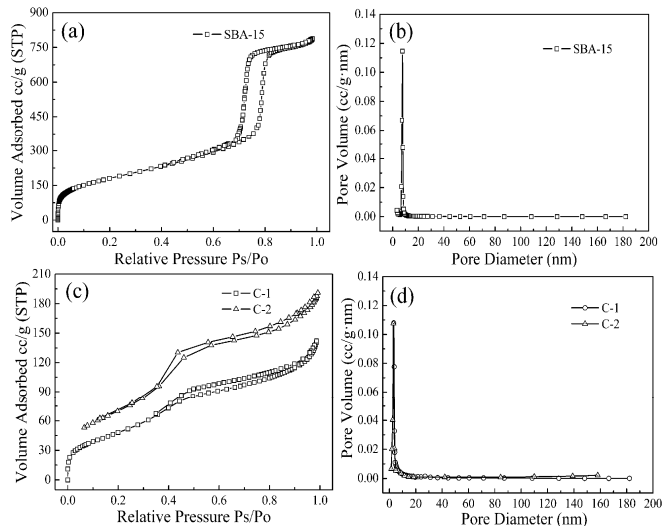

Fig. 2. Nitrogen sorption isotherms of SBA-15 (a), C-1 and C-2 (c) obtained at $77 \mathrm{~K}$ and the pore size distribution of SBA-15 (b), C-1 and C-2 (d) calculated by BJH method.

Fig. 2 displays the nitrogen adsorption/desorption isotherms and the pore size distribution curves of SBA-15 and as-prepared carbons. It can be seen that all samples display a type IV nitrogen adsorption/desorption isotherms (Fig. 2 (a, c)) and have centered pore size distribution (Fig. 2 (b, d)). Changes of weight ratio of M1:M2 do not affect the isotherms since C-1 and C-2 showed similar pore features. The isotherm of SBA-15 exhibits a distinct hysteresis of H1 type and is obvious different from that of $\mathrm{C}-1$ and $\mathrm{C}-2$. This may indicate that the melted mesophase pitch is not uniformly impregnated into the nanochannels of SBA-15 only by capillarity due to high apparent viscosity.

Table 1. Pore structure characterization of SBA-15, C-1 and C-2

\begin{tabular}{cccc}
\hline Sample & Pore size $[\mathrm{nm}]$ & Total pore volume $\left[\mathrm{cm}^{3} \mathrm{~g}^{-1}\right]$ & BET surface area $\left[\mathrm{m}^{2} \mathrm{~g}^{-1}\right]$ \\
SBA-15 & 7.6 & 1.2 & 658.1 \\
$\mathrm{C}-1$ & 4.5 & 0.3 & 256.0 \\
$\mathrm{C}-2$ & 3.3 & 0.2 & 177.4 \\
\hline
\end{tabular}

Table 1 shows the pore structure characterization of SBA-15, C-1 and C-2. With the increase dosage of mesophase pitch, pose size, total pore volume and BET surface area of carbons decrease. Therefore, pore structure of carbons could be effectively controlled by adjusting the dosage of mesophase pitch. Compared with porous carbons prepared by template carbonation using sucrose as carbon precursors [17], the pore volume and specific surface area of C-1 and C-2 prepared in this work decrease observably. This could be due to the different viscosity of precursors or relate to the graphitization characteristic of materials. Carbons prepared in this work could significantly reduce the formation of micropores [18].

Fig. 3 shows the Wide-angle X-ray diffraction (WAXD, a) and small-angle X-ray scattering (SAXS, b) patterns of $\mathrm{C}-1$ and $\mathrm{C}-2$. It is clear that $\mathrm{C}-1$ and $\mathrm{C}-2$ both have a similar crystalline structure. It is known that the height and width of (002) peak reflect the graphitization degree. C-1 and C-2 exhibit a partial graphitization, which is related to the oriented graphene layers and carbonization temperature. The (100), (110) and (200) diffraction peaks of SAXS patterns exhibit highly ordered long-range hexagonal spatial symmetric structures [19], providing further evidence for the replication of SBA-15 morphology of C-1 and C-2. 
Fig. 4 shows the first three charge-discharge curves at the current density of $35 \mathrm{~mA} \mathrm{~g}^{-1}$ between 0.005 and $2.0 \mathrm{~V}$ (vs. $\mathrm{Li}^{+} / \mathrm{Li}$ ). The curves present representative charge/discharge features of amorphous carbon [20,21]. The first charge-discharge capacities and coulombic efficiency of C-1 are $1050.5 \mathrm{mAh} \mathrm{g}^{-1}, 312.6 \mathrm{mAh} \mathrm{g}^{-1}$ and $29.8 \%$. Nevertheless, these of C-2 are $1050.2 \mathrm{mAh} \mathrm{g}^{-1}, 446.4$ $\mathrm{mAh} \mathrm{g}^{-1}$ and $42.5 \%$, respectively. C-1 and C-2 both possess large irreversible capacities, which mainly depend on their large specific surface area. The differences of the following two charged-discharged capacities may be attributed to the pore size and wall thickness of materials. Moreover, the first voltage plateau of $\mathrm{C}-1$ is much shorter than that of $\mathrm{C}-2$. Main reason is that the electrolyte is decomposed to form the solid electrolyte interface (SEI) films [22], and the film of C-2 is thicker and denser relative to $\mathrm{C}-1$. The SEI films could be verified by AC impedance spectra discussed hereafter.
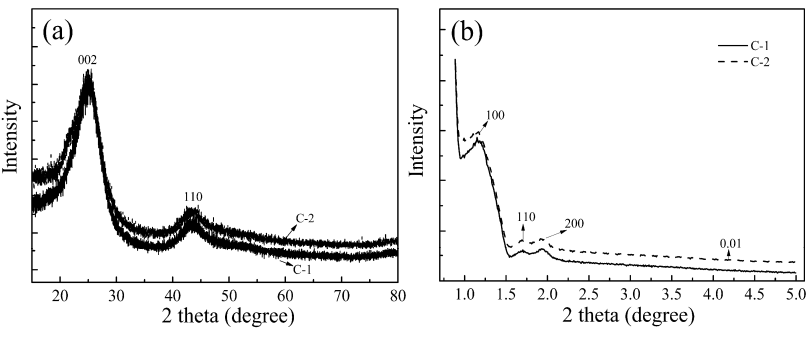

Fig. 3. WARD (a) and SAXS (b) patterns of C-1 and C-2.

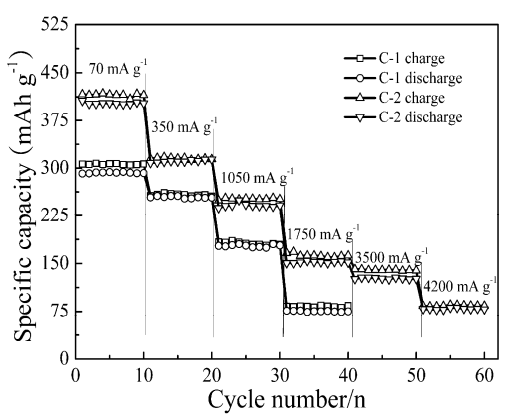

Fig. 5. Cycle performances of $\mathrm{C}-1$ and $\mathrm{C}-2$ at the different current density $\left(41-50^{\text {th }}\right.$ cycle)
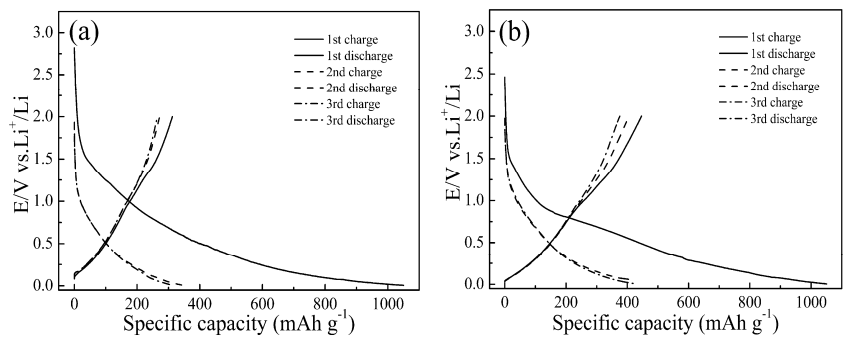

Fig. 4. The first three discharge-charge curves of C-1 (a) and C-2 (b): $35 \mathrm{~mA} \mathrm{~g}^{-1}$.

Fig. 5 illustrates the cycle performance of C-1 and C-2 at different current densities (41-50th cycle). The coulombic efficiencies of C-1 and C-2 maintain to be $98 \%$ after 50 cycles, indicating that as-prepared carbons exhibit excellent cycle performances. At low current density of $70 \mathrm{~mA} \mathrm{~g}^{-1}$, the reversible capacities and coulombic efficiencies of C-1and C-2 (the values is $293.5 \mathrm{mAh} \mathrm{g}$ and $403.3 \mathrm{mAh} \mathrm{g}^{-1}$, respectively) are close to those of graphite. When the current density increases to $1050 \mathrm{~mA} \mathrm{~g}^{-1}, \mathrm{C}-1$ and C-2 still have $50 \%$ reversible capacities. The results reveal that the carbons exhibit preferable anode properties for LIBs. At high current density of $3500 \mathrm{~mA} \mathrm{~g}^{-1}, \mathrm{C}-2$ exhibits a reversible capacity of $153.0 \mathrm{mAh} \mathrm{g}^{-1}$, whereas the capacity of $\mathrm{C}-1$ can not be measured, indicating that C-2 exhibits better preferable anode properties for LIBs than that of C-1.

The kinetics of electrode process of $\mathrm{C}-1$ and $\mathrm{C}-2$ are preliminarily studied by AC impedance spectra. Typical Nyquist complex plane impedance plots of C-1 and C-2 are presented in Fig. 6 (a). According to the previous publication [23,24], the high-frequency semicircle is attributed to the formation of SEI films on the surface of electrode and/or the diffusion resistance of $\mathrm{Li}^{+}$in SEI films; semicircle in medium-frequency region is ascribed to the impedance of charge-transfer process on electrode/electrolyte interface and the inclined line in the low frequency range corresponds to the Warburg diffusion, which associates with $\mathrm{Li}^{+}$diffusion process within electrode. From Fig. 6 (a), it can be clearly seen that, in high-frequency region the arc diameter for $\mathrm{C}-1$ is smaller than that of C-2, indicating the formation of thicker and denser SEI films in C-2 relative to $\mathrm{C}-1$. In medium-frequency 
region and the low frequency range, the arc length and slope for $\mathrm{C}-1$ and $\mathrm{C}-2$ have a little difference, an indication of the diverse charge-transfer impedance and $\mathrm{Li}^{+}$diffusion coefficient.

To further investigate the kinetic process of electrodes, the AC impendence spectra of C-1 and C-2 were modeled by the modified Randles equivalent circuit, which is presented in Fig. 6 (b) [25]. $R_{s}$ is the electrolyte resistance and $C P E_{1}$, and $R_{f}$ are the capacitance and resistance of the surface film formed on the electrodes, respectively. $\mathrm{CPE}_{2}$ and $\mathrm{R}_{\mathrm{ct}}$ denote the double-layer capacitance and charge-transfer resistance respectively. $Z_{\mathrm{w}}$ represents the Warburg impedance relating to diffusion of $\mathrm{Li}^{+}$into the bulk of electrodes. The exchange current density $\mathrm{i}_{0}$ can be calculated by Eq. 1 [26]:

$i_{0}=\frac{R T}{n F R_{\mathrm{ct}}}$

$\mathrm{Li}^{+}$diffusion coefficients $\mathrm{D}$ can be calculated from Eq. 2 [27]:

$Z_{\mathrm{w}}=\left(\frac{R T}{\sqrt{2} n^{2} F^{2} A}\right)\left(\frac{1}{\sqrt{D} C}\right) \quad\left(\omega>>\frac{2 D}{L^{2}}\right)$

where $\mathrm{R}$ is the gas constant, $\mathrm{T}$ the absolute temperature $(298.15 \mathrm{~K}), \mathrm{n}$ the number of transferred electrons, $\mathrm{F}$ the Faraday constant, $\mathrm{Z}_{\mathrm{w}}$ Warburg coefficient, A the cross sectional area between electrolyte and electrode, $\mathrm{D}$ the $\mathrm{Li}^{+}$diffusion coefficient and $\mathrm{C}$ the $\mathrm{Li}^{+}$concentration, $\omega$ the angular frequency of $\mathrm{AC}$ and $\mathrm{L}$ the thickness of electrodes.

The kinetic parameters of carbons are summarized in Table 2, which were obtained by ZsimpWin software fitting. The values of $\mathrm{R}_{\mathrm{s}}$ for $\mathrm{C}-1$ and $\mathrm{C}-2$ are almost unchanged, this is reasonable since $\mathrm{R}_{\mathrm{s}}$ is only related to electrolyte type. $\mathrm{R}_{\mathrm{f}}$ value of $\mathrm{C}-2$ is higher than that of $\mathrm{C}-1$. This is attributed to the formation of thicker and denser SEI film on the surface of C-2. The D value of C-2 is larger than that of $\mathrm{C}-1$ while their $\mathrm{R}_{\mathrm{ct}}$ and $\mathrm{i}_{0}$ are close, indicating that $\mathrm{C}-2$ as anode material for LIBs could exhibit higher electrochemical activity relative to $\mathrm{C}-1$.

Table 2. Kinetic parameters of C-1 and C-2

\begin{tabular}{cccccc}
\hline Sample & $R_{\mathrm{s}}\left[\Omega \cdot \mathrm{cm}^{2}\right]$ & $R_{\mathrm{f}}\left[\Omega \cdot \mathrm{cm}^{2}\right]$ & $R_{\mathrm{ct}}\left[\Omega \cdot \mathrm{cm}^{2}\right]$ & $i_{0}\left[10^{-4} \mathrm{~A} \mathrm{~cm}^{-2}\right]$ & $D\left[10^{-9} \mathrm{~cm}^{2} \mathrm{~s}^{-1}\right]$ \\
$\mathrm{C}-1$ & 9.17 & 59.57 & 21.79 & 11.79 & 5.98 \\
$\mathrm{C}-2$ & 9.99 & 72.03 & 27.45 & 9.36 & 8.47 \\
\hline
\end{tabular}

C-1 and C-2 possess tailored crystalline structure with graphene layers perpendicular to the axial of pore walls. The graphene layers provide convenient channels to rapidly reduce transmission distance for $\mathrm{Li}^{+}$insertion/detercation, and mesporous structure offer enough space for the free transportation of $\mathrm{Li}^{+}$inside prepared carbons. As a result, as-prepared carbons exhibit satisfactory anode performance as LIB anodes. Nevertheless, the diverse LIB properties mainly depend on the unimpressive difference of their meso-dimensions.

The pore walls of prepared carbons in this paper are similar to the platelet carbon nano-fibers. Many loops, parallel to the axial-direction of nano-fibers, are formed at the edge of graphene layers. These loops could prevent $\mathrm{Li}^{+}$intercalation/ deintercalation at the interlayers in carbons and not store energy. There is only a small difference for C-1 and C-2 in the pore wall thickness $(1.55 \mathrm{~nm}$ and 2.15 $\mathrm{nm}$, respectively). Meanwhile, $\mathrm{C}-1$ possesses higher specific surface area and larger pore size than $\mathrm{C}-2$. It is possible that the more loops could be formed on the surface of $\mathrm{C}-1$, which will hamper $\mathrm{Li}^{+}$ intercalation/deintercalation and result in the lower $\mathrm{D}$ value. Furthermore, it could be inferred from the parameters of pore structure that $\mathrm{C}-1$ with more loops will possess less percentage of carbon atoms per unit volume/mass for lithium storage than $\mathrm{C}-2$. These explanations could testify that as-prepared materials exhibit the diverse LIB anode properties only owing to the difference of pore size and wall thickness.

\section{Conclusions}

Two kinds of ordered mesoporous carbons (C-1 and C-2) with different precursor to template mass ratios are prepared using mesophase pitch as carbon precursors and SBA-15 sieves as template. As-prepared carbons possess the similar crystalline structure, different pore size and wall thickness, which significantly influence the anode properties for LIBs. The electrochemical tests show that C-1 
and C-2 both exhibit preferable specific capacity and specific power performance, but the anode properties of $\mathrm{C}-2$ is more excellent than $\mathrm{C}-1$ with higher specific surface area and thinner wall thickness. The comparative study of two ordered mesoporous carbons displays that the pore structure and wall thickness have a significant influence on anode properties for LIBs. Therefore, to obtain more excellent LIB anode performance, the pore size and wall thickness of mesoporous carbon materials should be optimized.

\section{Acknowledgements}

This work was supported by the National Natural Science Foundation of China (51272075, 50802028), Hunan Provincial Natural Science Foundation of China (10JJ2036) and Research Foundation of Education Bureau of Hunan Province, China (09A036).

\section{References}

[1] Y. G. Guo, J. S. Hu, L. J. Wan: Adv Mater, Vol. 20 (2008), p. 2878

[2] C. C. Li, X. M. Yin, L. B. Chen, Q. H. Li, T. H. Wang: J Phys Chem C, Vol.113 (2009), p. 13438

[3] I. Grigoriants, L. Sominski, H. Li, I. Ifargan, D. Aurbach, A. Gedanken: Chem Commun,Vol. 7 (2005), p. 921

[4] J. Fan, T. Wang, C. Yu, B. Tu, Z. Jiang, D. Zhao: Adv Mater, Vol. 16 (2004), p. 1432

[5] Su FB, Zhao XS, Wang Y, Z. C. Zhou, J. Y. Lee: J Phys Chem B, Vol. 109(2005), p. 20200

[6] W. Xing, P. Bai, Z. F. Li, R. J. Yu, Z. F. Yan, G. Q. Lu, L. M. Lu: Electrochim Acta, Vol. 51(2006), p. 4626

[7] K. T. Lee, J. C. Lytle, N. S. Ergang, A. Stein: Adv Funct Mater, Vol. 15 (2005), p. 547

[8] Y. Ren, A. R. Armstrong, F. Jiao, P. G. Bruce, J Am Chem Soc, Vol. 132 (2010), p. 996

[9] F. Jiao, P. G. Bruce: Adv Mater,Vol. 19 (2007), p. 657

[10] B. H. Clare, K. Efimenko, F. Genzer, N. L. Abbott: Chem Mater, Vol. 18(2006), p. 2357

[11] C. M. Noel, F. Giulieri, R. Combarieu, G. Bossis, A. M. Chaze: Colloids and Surfaces A: Physicochem Eng Aspects, Vol. 295(2007), p. 246

[12] K. Q. Jian, T. C. Truong, W. P. Hoffman, R. H. Hurt: Micropor Mesopor Mater, Vol. 108(2007), p. 143

[13] S. H. Yoon, S. Lim, S. H. Hong, K. Yokogawa: Carbon, Vol. 42(2004), p. 3087

[14] S. H. Yoon, C. W. Park, H. J. Yang, Y. Korai, I. Mochida, N. M. Rodriguez: Carbon, Vol. 42(2004), p. 21

[15] M. E. Sousa, C. Chan, K. Q. Jian, Y. M. Gao, R. H. Hurt, G. P. Crawford, N. Yang: Mol Cryst Liq Cryst, Vol. 435(2005), p. 767

[16] P. Hyldgaard, N. Jacobson, C. Ruberto, B. Razaznejad, B. I. Lundqvis: Comput Mater Sci, Vol. 33(2005), p. 356

[17] K. Bohme, W. D. Einicke, O. Klepel: Carbon, Vol. 43(2005), p. 1918

[18] H. F. Yang, Y. Yan, Y. Liu, F. Q. Zhang, R. Y. Zhang, Y. Meng, M. Li, S. H. Xie, B. Tu, D. Y. Zhao: J Phys Chem B, Vol. 108(2004), p. T17320

[19] M. Kaneda, T. Tsubakiyama, A. Carlsson, T. Ohsuna, O. Terasaki: J Phys Chem B,Vol.106(2002), p. 1256

[20] M. Winter, J. O. Besenhard, M.E. Spahr, P. Novák: Adv Mater Vol.10(1999) p. 725

[21] M. Endo, C. Kim, T. Fujino, K. Nishimura: Carbon, Vol. 38(2000), p. 183

[22] Y. K. Choi, K. Chung, Park SM, Sung YE, Park SM: J Power Sources, Vol. 104(2002), p. 132

[23] K. Fukuda, K. Kikuya, K. Isono, M. Yoshio: J Power Sources, Vol. 69(1997), p. 165

[24] S. Zhang, P. F. Shi: Electrochim Acta, Vol. 49 (2004), p. 1475

[25] S. B. Yang, J. P. Huo, H. H. Song, X. H. Chen: Electrochim Acta, Vol. 53 (2008), p. 2238

[26] S. Yang, H. Song, X. Chen: Electrochem Commun, Vol. 8 (2006), p. 137

[27] N.Takami, A. Satoh, T. Ohsaki, S. Kinetic: J Electrochem Soc, Vol. 142(1995), p. 371 